\title{
Characterization of Osteolytic, Osteoblastic, and Mixed Lesions in a Prostate Cancer Mouse Model Using ${ }^{18}$ F-FDG and ${ }^{18}$ F-Fluoride PET/CT
}

\author{
Wellington K. Hsu ${ }^{1}$, Mandeep S. Virk ${ }^{1}$, Brian T. Feeley ${ }^{1}$, David B. Stout ${ }^{2}$, Arion F. Chatziioannou ${ }^{2}$, and Jay R. Lieberman ${ }^{1}$ \\ ${ }^{I}$ Department of Orthopaedic Surgery, David Geffen School of Medicine at UCLA, Los Angeles, California; and ${ }^{2}$ Department of \\ Molecular and Medical Pharmacology, David Geffen School of Medicine at UCLA, Los Angeles, California
}

The combination of small-animal PET/CT scans and conventional imaging methods may enhance the evaluation of in vivo biologic interactions of murine models in the study of prostate cancer metastasis to bone. Methods: Small-animal PET/CT scans using ${ }^{18} \mathrm{~F}$-fluoride ion and ${ }^{18} \mathrm{~F}$-FDG coregistered with high-resolution small-animal CT scans were used to longitudinally assess the formation of osteoblastic, osteolytic, and mixed lesions formed by human prostate cancer cell lines in a severe combined immunodeficient (SCID) mouse tibial injection model. These scans were correlated with plain radiographs, histomorphometry, and soft-tissue measurements. Results: Smallanimal PET/CT scans were able to detect biologic activity of cells that induced an osteoblastic lesion $2 \mathrm{wk}$ earlier than on plain radiographs. Furthermore, both the size and the activity of the lesions detected on PET/CT images significantly increased at each successive time point $(P<0.05)$. ${ }^{18} \mathrm{~F}$-FDG lesions strongly correlated with soft-tissue measurements, whereas ${ }^{18} \mathrm{~F}$-fluoride ion activity correlated with bone volume measured on histomorphometric analysis $(P<0.005)$. Osteolytic lesions were successfully quantified using small-animal $\mathrm{CT}$, whereas lesion sizes measured on ${ }^{18} \mathrm{~F}-\mathrm{FDG}$ PET scans also strongly correlated with soft-tissue tumor burden $(P<0.05)$. In contrast, for mixed lesions, ${ }^{18} \mathrm{~F}$-fluoride ion and ${ }^{18} \mathrm{~F}$-FDG PET/CT scans detected only minimal activity. Conclusion: ${ }^{18} \mathrm{~F}-\mathrm{FDG}$ and ${ }^{18} \mathrm{~F}$-fluoride ion PET/CT scans can be useful tools in characterizing pure osteolytic and osteoblastic lesions induced by human prostate cancer cell lines. The value of this technology needs further evaluation to determine whether these studies can be used effectively to detect more subtle responses to different treatment regimens in animal models.

Key Words: PET/CT; small-animal imaging; prostate cancer; osteoblastic lesion; osteolytic lesion

J Nucl Med 2008; 49:414-421

DOI: 10.2967/jnumed.107.045666

\footnotetext{
Received Jul. 26, 2007; revision accepted Nov. 27, 2007.

For correspondence or reprints contact Jay R. Lieberman, MD, Department of Orthopaedic Surgery, The Musculoskeletal Institute, University of Connecticut Health Center. 263 Farmington Ave., Farmington, Connecticut 06030-5456.

E-mail: jlieberman@uchc.edu

COPYRIGHT @ 2008 by the Society of Nuclear Medicine, Inc.
}

$\mathbf{P}$ rostate adenocarcinoma affects millions of men annually and is the leading newly diagnosed cancer in American men (1). Bone metastases from prostate cancer usually form osteoblastic lesions but may also be associated with mixed or osteolytic lesions as well $(2,3)$. Transgenic and severe combined immunodeficient (SCID) mouse models have been essential in enhancing our understanding of the biologic interactions between human prostate cancer cells and bone $(4,5)$. However, these animal models have some limitations. First, the traditional outcome measures, such as plain radiographs, histology, and histomorphometry, do not measure dynamic biologic activity, such as cellular metabolism. Second, plain radiographs provide only a qualitative assessment of tumor activity and do not identify subtle changes in bone formation or loss. Third, studies that use histologic specimens are subject to potential sampling limitations that can lead to misleading results (6).

PET is a well-established, noninvasive imaging modality to evaluate metabolic activity through the uptake of radioactive tracers in tissue. Historically, clinical PET and CT scanners were hindered by low-resolution capabilities not suitable for animal models of disease (7). The development of high-resolution combined PET/CT scanners dedicated to small-animal imaging allows the critical study of bone (8-10).

Different radioactive tracers labeled with positronemitting radionuclides can be delivered systemically, which localize in living tissue. For example, ${ }^{18} \mathrm{~F}$-fluoride ion has been shown to deposit preferentially at the surface of bone, where the greatest activity of remodeling and turnover are seen $(11) .{ }^{18} \mathrm{~F}$-Fluoride ion exchanges with hydroxyl groups on hydroxyapatite to form fluoroapatite, which subsequently incorporates into bone matrix (12). ${ }^{18} \mathrm{~F}-\mathrm{FDG}$, which correlates with cellular glucose metabolism, is the most widely used radioactive tracer in oncology and is often used in whole-body scanning to detect distant metastases (13). ${ }^{18} \mathrm{~F}-$ FDG is considered a valuable adjunct to existing imaging methods in an increasing number of clinical oncologic applications, such as metastatic adenocarcinoma and musculoskeletal sarcomas (14-16). Although metastatic prostate 
cancer in humans traditionally exhibits relatively low uptake of ${ }^{18} \mathrm{~F}-\mathrm{FDG}$, several studies have demonstrated its utility as a promising prognostic measure for patient survival $(17,18)$.

Plain radiographs reveal the late manifestations of tumor cell activity, including osteolytic or osteoblastic lesions. However, demonstration of localized metabolic activity from high-resolution PET/CT images could lead to prospective assessments of tumor growth and its effect on bone. Furthermore, the ability to quantify the osteoblastic and osteolytic components of a prostate cancer lesion in vivo could be advantageous in providing an objective evaluation of different therapeutic regimens. Animals can potentially be followed longitudinally throughout the course of a study, obviating the need for histologic specimens at multiple time points. Because of its high-resolution imaging capabilities, we postulated that microPET/CT could aid in the characterization of osteolytic, osteoblastic, and mixed human prostate cancer lesions in a murine tibial injection model.

\section{MATERIALS AND METHODS}

\section{Cell Lines}

Human prostate cancer cell lines PC-3, LAPC-9, and C42B were used in this study. LAPC-9 was derived from a human metastatic lesion to bone and has been reported in prior studies (19-22). LAPC-9 produces a purely osteoblastic lesion when surrounded by bone. PC-3 (American Type Culture Collection) has been demonstrated to form a purely osteolytic lesion when implanted into bone (23). The C42B cell line (gift of Irvin Chung, $\mathrm{PhD}$, Emory University) is derived from LNCaP cells (24) and has been shown to result in a mixed osteoblastic and osteolytic lesion when placed into bone. Both PC-3 and C42B cells can be maintained in cell culture for an extended period of time, whereas LAPC9 cells can be kept in culture for only $48 \mathrm{~h}$. PC-3 and C42B cells were cultured in Iscove's medium (Irvine Scientific) supplemented with $10 \%$ fetal bovine serum (FBS) and $1 \%$ glutamine and maintained at $37^{\circ} \mathrm{C}$ in a humidified atmosphere with $5 \% \mathrm{CO}_{2}$.

\section{Cell Suspension Protocol}

SCID mice with subcutaneous LAPC-9, PC-3, or C42B tumors were anesthetized (100 mg ketamine/kg body weight, $10 \mathrm{mg}$ xylazine/kg body weight) and then euthanized. Dorsal skin was then shaved and prepped under standard sterile conditions using $70 \% \mathrm{EtOH}$ and povidone iodine. The subcutaneous tumor was resected and minced in phosphate-buffered saline (Invitrogen Life Technologies) using a sterile scalpel blade. The solution was then centrifuged at 1,400 rpm for $5 \mathrm{~min}$ at room temperature. After aspiration of the supernatant, the residual pellet was resuspended in a $0.1 \%$ pronase E/Iscove's medium solution that was previously filtered using a $0.22-\mu \mathrm{m}$ Steriflip filter (Millipore Corp.). The cell suspension was slowly shaken for $18 \mathrm{~min}$ at room temperature, kept in ice for $2 \mathrm{~min}$, and subsequently passed through a $70-\mu \mathrm{m}$ cell strainer. The mixture was then centrifuged at $1,300 \mathrm{rpm}$ for $5 \mathrm{~min}$ at room temperature. Supernatant was aspirated and the pellet was resuspended in Iscove's medium with $15 \%$ FBS and $1 \%$ glutamine and plated in 10-mL culture dishes. Cells were maintained at $37^{\circ} \mathrm{C}$ in a humidified incubator with $5 \% \mathrm{CO}_{2}$.

\section{Animals}

Eight- to 10-wk-old male SCID mice were maintained under pathogen-free conditions after protocol approval by the UCLA (University of California, Los Angeles) Chancellor's Animal Research Committee (ARC). Animals were housed in accordance with the UCLA Department of Laboratory Animal Medicine guidelines and were provided food and water ad libitum.

\section{Surgical Technique}

Five microliters of Matrigel (BD Biosciences) were added to single-cell suspensions of LAPC- 9 or PC-3 cells to form a $10-\mu \mathrm{L}$ mixture containing $1 \times 10^{5}$ cells. Mice were administered a loading dose of intraperitoneal anesthesia (100 mg ketamine/kg body weight, $10 \mathrm{mg}$ xylazine/kg body weight). Maintenance anesthesia was then provided using continuous delivery of $1 \%$ isoflurane through a nose cone. Left hind limbs were shaved and prepped under standard sterile conditions using $70 \% \mathrm{EtOH}$ and povidone iodine. Using a number 15 scalpel blade, a 2- to 3-mm longitudinal incision over the midpatellar region was made. An arthrotomy was then performed using the tip of the blade. A 27-gauge, 0.5-in needle on a tuberculin syringe containing the cell line/matrigel mixture was inserted through the middle of the tibial plateau and advanced $\sim 3$ $\mathrm{mm}$ into subchondral bone. Injection of the cell suspensions was performed slowly with minimal resistance. A 2-layer skin closure was performed with a 5-0 Vicryl suture (Ethicon Inc.). The time until animal sacrifice was chosen for each prostate cancer cell line on the basis of previously reported rates of growth and the size limitations of tumors established by the UCLA ARC. For PC-3 tumors, animals were sacrificed at 2-, 4-, and 6-wk time points; for LAPC-9 cells, animals were sacrificed at 4-, 6-, and 8-wk time points; and for $\mathrm{C} 42 \mathrm{~B}$ tumors, 4-, 8-, and 12-wk time points were used. Animals were sacrificed before the soft-tissue tumor burden reached $1.5 \mathrm{~cm}$ in diameter. These measurements were chosen in accordance with regulations enforced by the ARC. At each time point, animals were sacrificed, radiographed with a Faxitron (Field Emission Corp.), imaged using standardized microPET and microCT protocols, and had their tumor harvested for soft-tissue measurement and histomorphometric analysis. Tumor burden calculations were performed with the function:

$$
\text { Volume }=(\text { length })(\text { width })^{2} \cdot 0.52(25)
$$

Three animals were used at each time point for both ${ }^{18} \mathrm{~F}-\mathrm{FDG}$ and ${ }^{18}$ F-fluoride ion tracers for all cell lines.

\section{Tracer Preparation}

Fluoride ion was produced using ${ }^{18} \mathrm{O}$-water and proton bombardment using a RDS cyclotron (Siemens Preclinical Solutions). ${ }^{18} \mathrm{~F}$-Fluoride ion was produced at specific activities of $\sim 37 \mathrm{TBq} / \mathrm{mmol}(\sim 1,000 \mathrm{Ci} / \mathrm{mmol})$ using a method reported by Wieland et al. (26). ${ }^{18} \mathrm{~F}-\mathrm{FDG}$ was synthesized by the method described by Hamacher et al. (27) at specific activities of $\sim 185$ $\mathrm{GBq} / \mathrm{mmol}(\sim 5,000 \mathrm{mCi} / \mathrm{mmol})$.

\section{PET Protocol}

Using an ARC-approved isolated imaging chamber that provided continuous delivery of $1 \%-3 \%$ isoflurane anesthesia in oxygen and temperature control at $36^{\circ} \mathrm{C}(28)$, all animals underwent PET scans at the appropriate time points. Mice were injected with $\sim 9.25 \mathrm{MBq}(\sim 250 \mu \mathrm{Ci})$ of ${ }^{18}$ F-fluoride ion via tail vein using a 27 -gauge, 0.5 -in needle on a tuberculin syringe. Bladders 
were manually expressed $5 \mathrm{~min}$ before imaging to decrease background signal. Animals were positioned in a multimodality, portable isolated bed system consisting of a polymethyl methacrylate chamber with anesthesia ports and raised platform (29). Whole-body scans were performed with a 10-min acquisition time using a microPET FOCUS 220 system (Siemens Preclinical Solutions). Immediately afterward, a noncontrast-enhanced microCT study using a microCAT II (Siemens Preclinical Solutions) imaging system was used to scan animals with a 30-min acquisition time. Mice were then placed in a recovery chamber and monitored carefully for respiratory difficulties. PET scan images were reconstructed using filtered backprojection and an iterative 3-dimensional reconstruction algorithm (maximum a posteriori [MAP]) (30). microCT images were created using Fledkamp reconstruction at $200-\mu \mathrm{m}$ resolution. microPET and microCAT images were then overlaid for analysis for use with AMIDE software (31). Reproducibility of the coregistration of both scans for the mouse, when using this imaging technique, has been found to be acceptable (28). Whole-body scans using the same acquisition protocol with 9.25 $\mathrm{MBq}(250 \mu \mathrm{Ci})$ of ${ }^{18} \mathrm{~F}-\mathrm{FDG}$ were performed for different mice at the same weekly intervals.

\section{Quantitative Analysis}

${ }^{18}$ F-Fluoride and ${ }^{18}$ F-FDG PET and CT data were analyzed and quantified by AMIDE (A Medical Image Data Examiner), version 0.7.154 (31). Plain anteroposterior radiographs were superimposed onto reconstructed PET/CT images, and tibial injection sites were identified. Two separate protocols were used to analyze PET images to quantify tumor size and activity.

To quantify the size of the lesion in the proximal tibias, regions of interest (ROIs) were drawn using a 3-dimensional isocontour ROI tool identifying voxels of similar signal intensity. Manual selection of a voxel within the site of tumor uptake was confirmed by 2 independent reviewers. The isocontour tool then drew a 3-dimensional ROI based on voxels that exhibited signal intensity either greater or equal to the manually chosen voxel. Final ROIs were checked to ensure that signal uptake within the proximal tibia was included. Because background radioactivity is seen around physeal growth plates, ROIs using similar isocontour values over the contralateral proximal tibia were measured to control for background radioactivity. Tumor size was then calculated by subtracting control tibial ROIs from those of tibias with tumor cells. The AMIDE data analysis tool was used to calculate ROI sizes (in $\mathrm{mm}^{3}$ ).

Tumor activity was quantified using manually drawn ROI boxes $(34 \times 20 \times 10 \mathrm{~mm})$ over bilateral proximal tibias that were 3-dimensionally reconstructed to confine all discernible signal uptake. AMIDE data analysis tools were then used to calculate median signal intensity of the voxels contained in the ROI box. This process was repeated for both the control and the experimental group tibias. The difference in uptake between tibias was reported as a percentage increase of absolute uptake in the tibia containing the prostate cancer lesion. To assess the potential effect of the injection on the PET scan results, sham surgeries were performed in 3 SCID mice in which only Matrigel was injected into the proximal tibia. PET/CT images of these animals revealed no measurable difference in tracer uptake compared with control animals 2 wk after surgery (data not shown).

For PC-3 and C42B tumors, microCT images were used to identify osteolytic lesions in comparison with the contralateral control tibia. ROIs were drawn and reconstructed over bilateral tibias using AMIDE and a standardized isocontour value used for cortical bone $(\sim 2.20)$. Osteolytic lesion size was measured by subtracting tumor tibial ROIs from control tibial ROIs. Data at each time point for PET/CT images were compiled and analyzed by 2 independent reviewers.

\section{Histologic and Histomorphometric Analysis}

After animal sacrifice, tibias from each limb were subjected to histologic analysis. Lower extremities were amputated en bloc at the level of the distal femur. Specimens were then fixed in $10 \%$ buffered formalin, followed by decalcification in $10 \%$ ethylenediaminetetraacetic acid (EDTA) solution for $2 \mathrm{wk}$ at room temperature with mechanical stirring. Sagittal sections were cut manually and stained with hematoxylin and eosin, Orange G, and tartrate-resistant acid phosphatase activity. An Olympus imaging system was used to perform histomorphometric analysis with BIOQUANT software (BIOQUANT Image Analysis Corp.).

\section{Statistical Analysis}

For each experiment, ROIs and tumor sizes were reported as mean \pm SD. Soft-tissue tumor measurements and PET scan tumor-size quantifications were compared using standard logistic regression methods. Statistical significance was considered present when $P<0.05$. Data obtained from ROI calculations and histomorphometric analysis were compared using 2-way ANOVA.

\section{RESULTS}

For all 3 human prostate cancer cell lines, lesion size and signal intensity were quantified using ${ }^{18} \mathrm{~F}$-fluoride ion and ${ }^{18} \mathrm{~F}$-FDG PET/CT scans, and soft-tissue tumor burden was measured at each time point. In addition, for those cell lines with an osteoblastic component (LAPC-9 and C42B), histomorphometric analysis was used as an outcome measure. For osteolytic lesions (PC-3 and C42B), microCT was used to quantify lesion size using contralateral tibia volume. Because newly formed bone formed from LAPC-9 and $\mathrm{C} 42 \mathrm{~B}$ lesions closely approximated signal intensity of host bone, the resolution capabilities of microCT were not sufficient to distinguish an osteoblastic lesion from native tibia.

Lesion size was quantified on ${ }^{18}$ F-fluoride ion PET/CT scans for LAPC-9 cells, which form pure osteoblastic lesions. Statistically significantly larger lesions were found at each time point using ${ }^{18}$ F-fluoride ion imaging $(P<$ 0.05) (Fig. 1). Qualitatively, signal uptake was confined primarily within the cortical bone of the tibia and extended distally from the injection site in larger lesions (Fig. 2). Furthermore, statistically significantly higher signal intensity was measured at each time point using ${ }^{18} \mathrm{~F}$-fluoride ion $(P<0.05)$ (Table 1). Corresponding PET/CT images, plain radiographs, and histologic specimens were evaluated for the presence of an osteoblastic lesion at the 4-, 6-, and 8-wk time points (Fig. 2). Although osteoblastic lesions could not be identified on plain radiographs until the 6-wk time point, PET/CT images detected tracer uptake as early as $4 \mathrm{wk}$ after tumor implantation. Furthermore, osteoblastic activity measured on PET/CT scans was well visualized using qualitative assessments of reconstructed images (Fig. 2). 


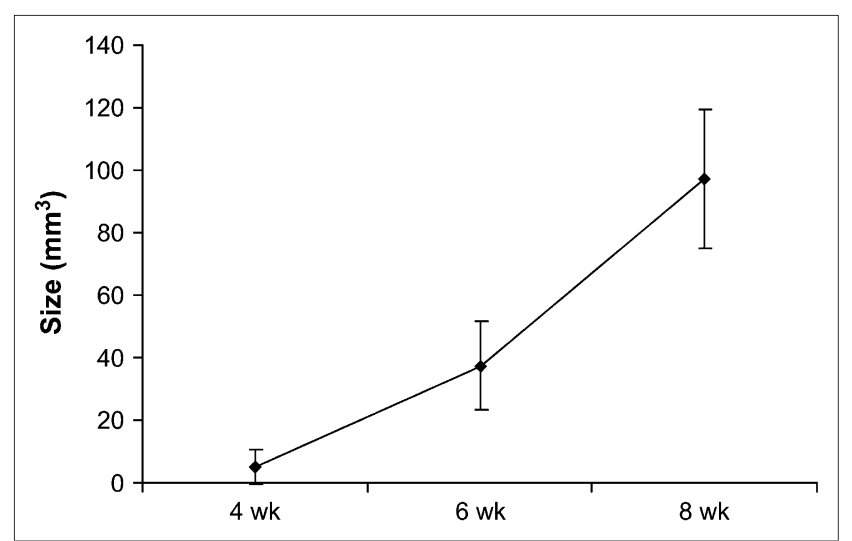

FIGURE 1. ${ }^{18}$ F-Fluoride ion PET/CT scans, used to measure osteoblastic lesion size, demonstrate increased uptake in LAPC-9 tumors at each time point. ROI sizes were statistically significantly greater at each successive interval $(P<0.05)$. The PET scan allows for quantification of the size of the osteoblastic lesion.

Median signal intensity measured for all animals strongly correlated with bone volume measured with histomorphometric analysis $\left(R^{2}=0.71\right)$ (Fig. 3).

LAPC-9 tumors were also evaluated using ${ }^{18} \mathrm{~F}-\mathrm{FDG}$ PET/CT scans in different animals at the same time points. Both lesion size and localized signal intensity increased significantly at each time point on ${ }^{18} \mathrm{~F}$-FDG images $(P<$ 0.05) (Fig. 4) (Table 1). Lesion sizes measured on PET images were compared with soft-tissue measurements of harvested tumors using standardized logistic regression analysis with evidence of strong statistical correlation $\left(R^{2}=0.92\right)$. Qualitatively, signal uptake was found around and within the periphery of the soft-tissue shadow seen on CT and plain radiography, with the most intense signal measured within bone (Fig. 4).

PC-3 tumors, which form pure osteolytic lesions, were also assessed using both ${ }^{18} \mathrm{~F}$-fluoride ion and ${ }^{18} \mathrm{~F}$-FDG PET/CT scans. Although PC-3 tumors exhibited early ${ }^{18} \mathrm{~F}-$ fluoride ion uptake at the 2- and 4-wk time points, size measurements were not statistically significantly different at any time point (data not shown). In each animal, as the osteolytic lesion expanded, less host bone was available for deposition of ${ }^{18} \mathrm{~F}$-fluoride ion. As a result, at the 6-wk time point, no radioactivity was seen within the ROI (data not shown). ${ }^{18}$ F-FDG PET/CT scans at the 2-, 4-, and 6-wk time points revealed statistically significantly increased lesion size and specific uptake values at each time point $(P<0.04)$ (Table 2). Similar to that of LAPC-9 tumors, lesion sizes were compared with soft-tissue measurements and found to correlate significantly on logistic regression analysis $\left(R^{2}=0.86\right)$ (graph not shown).

The osteolytic lesions induced by PC-3 cells were assessed using plain radiographs, microPET/CT images, and histologic specimens at each time point (Fig. 5). Plain radiographs revealed progressive bone loss beginning at the 2-wk time point. microCT allowed for quantification of

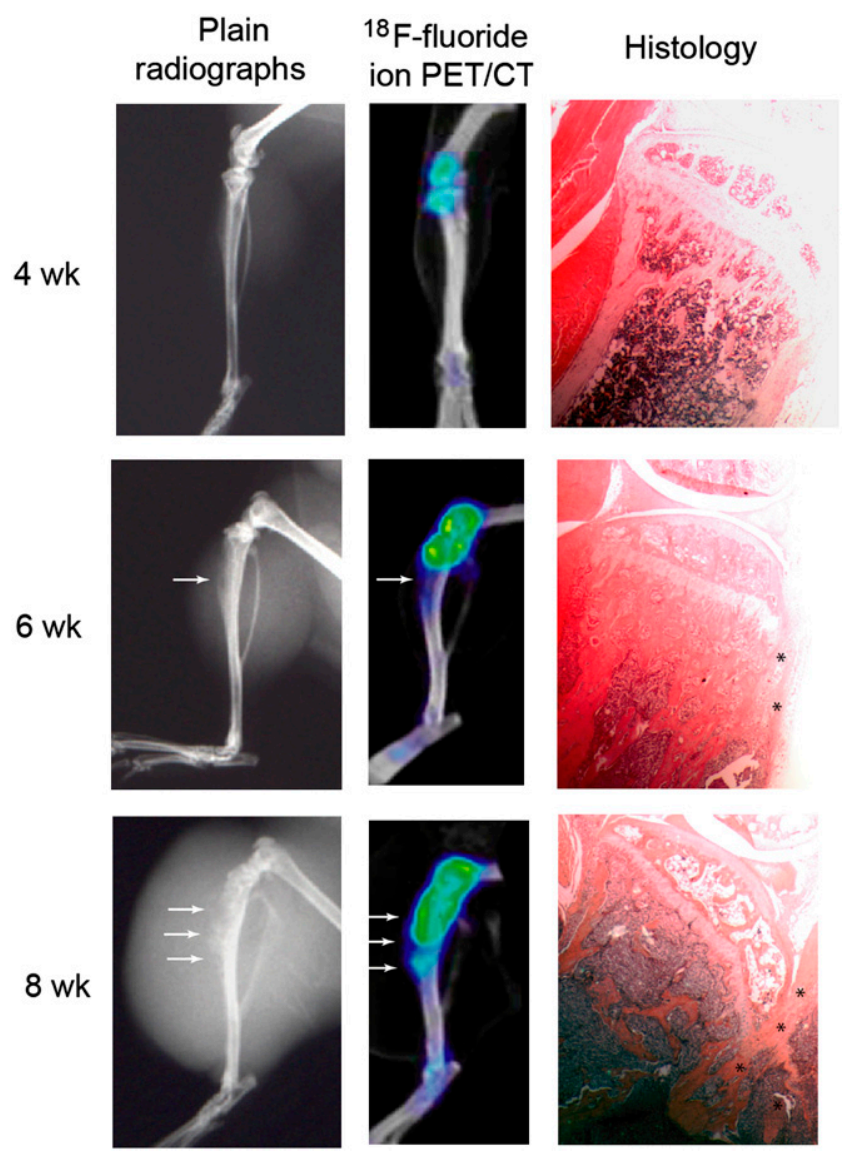

FIGURE 2. Plain radiographs, corresponding PET/CT images, and histologic specimens of representative LAPC-9 tumors using ${ }^{18}$ F-fluoride ion at 4-, 6-, and 8-wk time points. Although bone formation is visible on plain radiographs at the 6- and 8 -wk time points (arrows), a progressive increase of ${ }^{18} \mathrm{~F}$-fluoride ion uptake indicating osteoblastic activity is well visualized on successive PET images beginning at 4 wk after tumor cell injection (arrows). Increasing signal intensity on PET scans corresponded to new bone formation seen on high-power histologic analysis (asterisks).

bone loss at each successive time point using the contralateral tibia as a control value. Osteolytic lesion size was significantly greater at each time point $(P<0.05)$ (Fig. 6). Furthermore, bone loss seen on imaging correlated with osteoclastic activity and tumor cell infiltration on histologic analysis and increased ${ }^{18} \mathrm{~F}$-FDG uptake in the surrounding soft tissues (Fig. 5).

C42B tumors, which characteristically produce mixed osteolytic and osteoblastic lesions, were evaluated with

TABLE 1

Percentage Increase in Tracer Uptake in LAPC9 Cells

\begin{tabular}{cccc}
\hline \multirow{2}{*}{ Tracer } & \multicolumn{3}{c}{ Increase in tracer uptake (\%) } \\
\cline { 2 - 4 } & $4 \mathrm{wk}$ & $6 \mathrm{wk}$ & $8 \mathrm{wk}$ \\
\hline${ }^{18} \mathrm{~F}-$ Fluoride ion & $7.9 \pm 5.7$ & $42.5 \pm 13.3$ & $94.4 \pm 53.4$ \\
${ }^{18} \mathrm{~F}-\mathrm{FDG}$ & $13.8 \pm 14.6$ & $70.3 \pm 33.6$ & $224.5 \pm 248.3$ \\
\hline
\end{tabular}




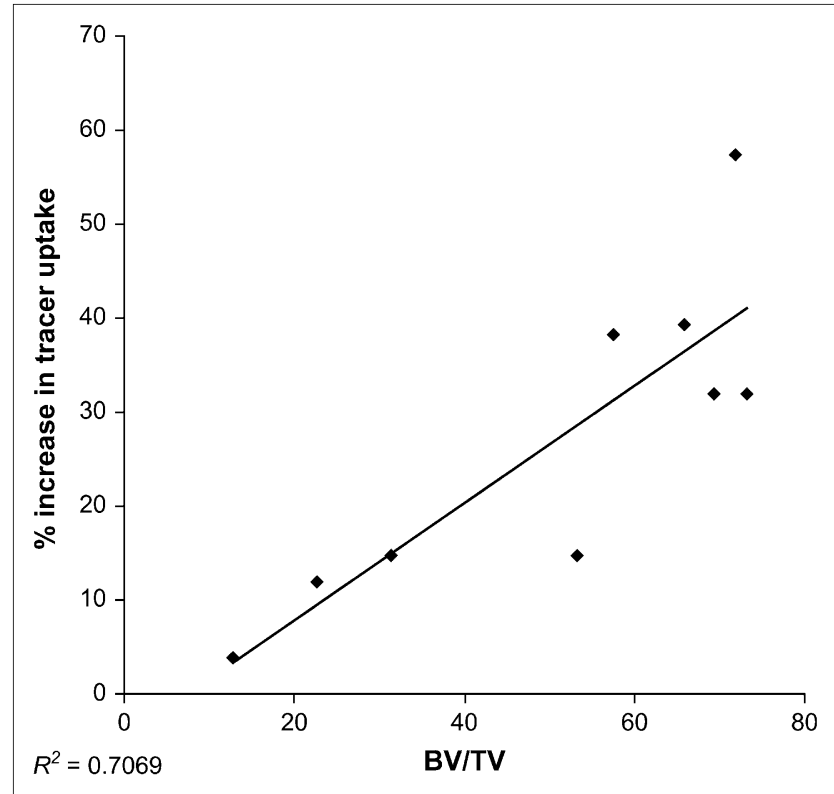

FIGURE 3. Analysis of PET scans after intratibial injections of LAPC-9 cells. Logistic regression analysis comparing localized median signal intensity seen on ${ }^{18} \mathrm{~F}$-fluoride ion PET scans and bone volume measured using histomorphometric analysis was performed. Tracer uptake was reported as a percentage increase in median signal intensity in relation to the control, uninjected tibia. A strong correlation was established between data points $(P=0.005)\left(R^{2}=0.71\right)$. BV = bone volume, $\mathrm{TV}=$ tissue volume.

${ }^{18} \mathrm{~F}$-fluoride ion and ${ }^{18} \mathrm{~F}-\mathrm{FDG}$ PET/CT scans at 4-, 8-, and 12 -wk time points. Minimal ${ }^{18} \mathrm{~F}$-fluoride ion uptake measured at the 3 successive intervals was not statistically significantly different from each other, and minimal osteoblastic changes were noted on plain radiographs (Fig. 7). C42B tumors were seen to have a mixed osteolytic and osteoblastic appearance on plain radiographs; however, this activity was not correlated on ${ }^{18} \mathrm{~F}$-fluoride ion PET/CT scans. ${ }^{18} \mathrm{~F}-\mathrm{FDG}$ PET revealed progressively increasing signal intensity at each time point; however, lesion size calculations were consistently smaller than the soft-tissue tumor burden measurements. Furthermore, tumor sizes derived from PET images were widely variable. Whereas C42B ROIs measured $283 \pm 181 \mathrm{~mm}^{3}$ at the 12 -wk time point, soft-tissue tumor burdens of the same lesions were calculated at $1,279 \pm 621 \mathrm{~mm}^{3}$. microCT was used to measure the size of the osteolytic lesion; however, no significant differences were seen between each successive time point (data not shown).

\section{DISCUSSION}

Animal models of bone metastasis are critical in the advancement of our understanding of the molecular mechanisms associated with the development of bone lesions and the identification of potential treatment regimens for metastatic prostate cancer. Plain radiographs, histologic analysis, and histomorphometric analysis are excellent tools to

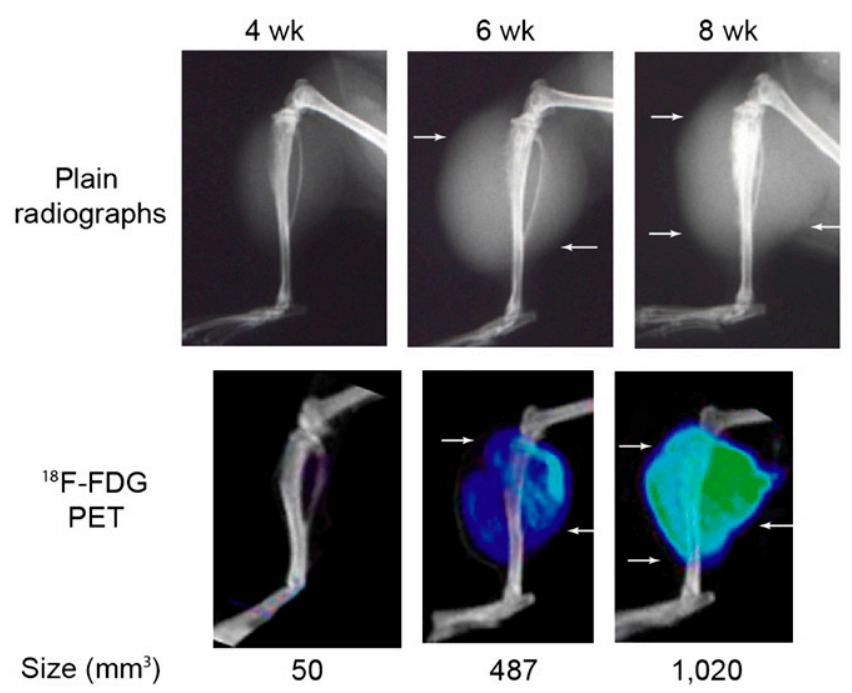

FIGURE 4. Plain radiographs and corresponding PET/CT images of a representative LAPC- 9 tumor using ${ }^{18} \mathrm{~F}-\mathrm{FDG}$ at $4-, 6-$, and 8 -wk time points. A progressive increase of ${ }^{18} \mathrm{~F}-\mathrm{FDG}$ uptake on PET images is pervasive where soft-tissue shadows are visible on plain radiographs (arrows). LAPC-9 tumors at the 8-wk time point show the strongest signal intensity (green), with tracer uptake seen at the outermost periphery of the lesion. Using quantitative analysis, lesion size (measured in $\mathrm{mm}^{3}$ and labeled below each corresponding tumor) can be calculated from PET images.

analyze the tumor manifestations in bone; however, the advent of high-resolution imaging systems suitable for small animals, such as microPET/CT scans $(8,32)$, offers the capability of noninvasive in vivo quantification of critical biologic processes.

The goal of this study was to determine whether microPET/CT scans could be a useful adjunct to the traditional tools used to study the biologic interactions between tumor cells in bone. LAPC-9 cells produce pure osteoblastic lesions, and PET using ${ }^{18} \mathrm{~F}-\mathrm{FDG}$ and ${ }^{18} \mathrm{~F}$-fluoride ion detected lesions with significantly greater size and biologic activity at each successive time point. These data were compared with well-accepted parameters to measure tumor activity: direct measurements of soft-tissue lesion size and histomorphometric indices of bone volume. Strong correlations were established between lesion size and soft-tissue burden as well as median signal intensity and bone volume measured on histomorphometric analysis, validating these quantification methods. Other studies have shown similar correlations between ${ }^{18} \mathrm{~F}$-fluoride ion PET scans and bone histomorphometry indices of bone formation $(33,34)$. Fur-

TABLE 2

Percentage Increase in Tracer Uptake in PC-3 Cells

\begin{tabular}{cccc}
\hline & \multicolumn{3}{c}{ Increase in tracer uptake (\%) } \\
\cline { 2 - 4 } Tracer & $2 \mathrm{wk}$ & $4 \mathrm{wk}$ & $6 \mathrm{wk}$ \\
\hline${ }^{18} \mathrm{~F}-\mathrm{FDG}$ & $0.8 \pm 33.9$ & $57.8 \pm 3.2$ & $84.4 \pm 6.4$ \\
\hline
\end{tabular}



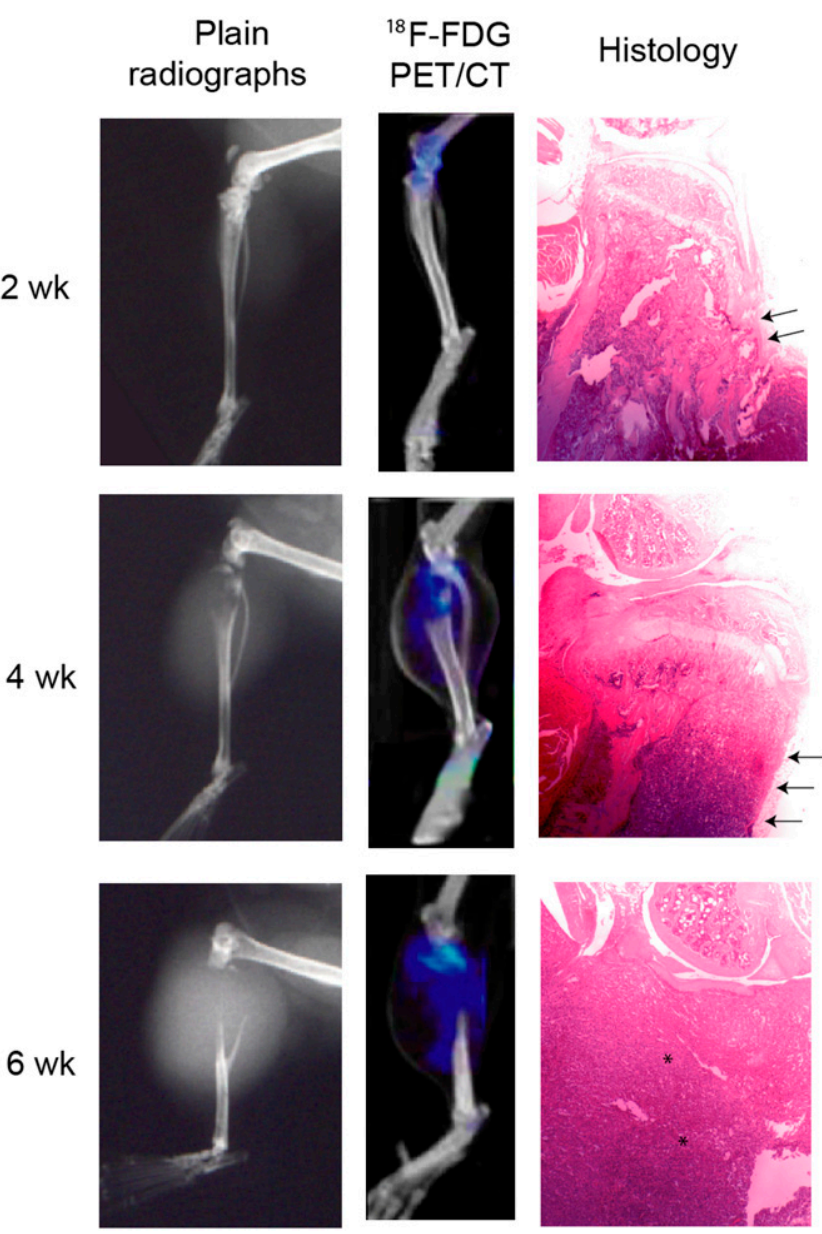

FIGURE 5. Plain radiographs and corresponding microPET/ CT images using ${ }^{18} \mathrm{~F}-\mathrm{FDG}$ of a representative PC-3 tumor at 2-, 4-, and 6-wk time points. A progressively larger osteolytic lesion is discernible on both plain radiographs and microCT images at successive time intervals, where the absence of proximal tibial bone is seen. In addition, increased ${ }^{18} \mathrm{~F}-\mathrm{FDG}$ uptake was found to localize in soft tissues surrounding the osteolysis at each time point. Using AMIDE data analysis tools on microCT scans, osteolytic lesion volume can be measured by comparing values with contralateral control tibia. Osteolysis seen on microCT images corresponded to histologic analysis, which revealed cortical disruption (arrows) and progressive infiltration of tumor cells (asterisks).

thermore, ${ }^{18} \mathrm{~F}$-fluoride ion uptake was noted 4 wk after LAPC-9 cell injection, when no lesion was visible on plain radiographs.

For pure osteolytic lesions induced by PC-3 cells, microCT was successfully used to quantify lesion size based on bone loss. Significantly larger areas of osteolysis were measured at each successive time point. ${ }^{18} \mathrm{~F}-\mathrm{FDG}$ is not specific to tumor metabolism and can accumulate in metabolically active inflammatory cells; however, the usefulness of ${ }^{18} \mathrm{~F}$-FDG in human prostate adenocarcinoma is unclear. Some investigators have reported low uptake of ${ }^{18} \mathrm{~F}$-FDG in patients with prostate cancer, whereas in other studies, ${ }^{18}$ F-FDG PET/CT scans have been used to identify osseous and soft-tissue metastasis from prostate adenocar-

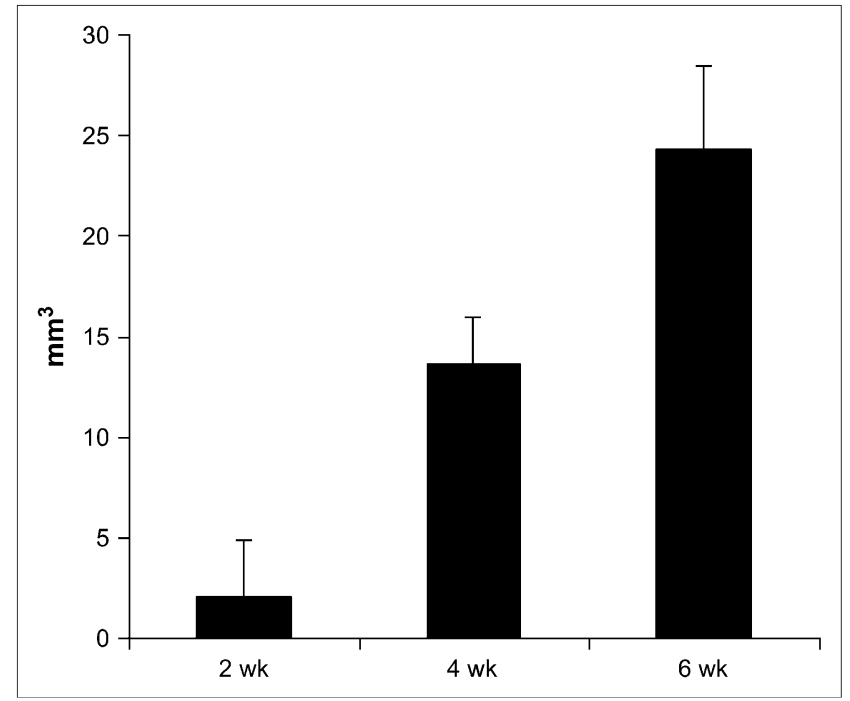

FIGURE 6. Osteolytic lesion size measured in PC-3 tumors using microCT at 2-, 4-, and 6-wk time points. Using contralateral tibia volume, bone loss was quantified using differences in voxel signal intensity. Statistically significantly larger lesions were measured at each successive time point $(P<0.05)$.

cinoma $(17,18,35-37)$. Our data demonstrated early tracer uptake $(2 \mathrm{wk})$ and a strong correlation between soft-tissue tumor burden measurements with ${ }^{18} \mathrm{~F}$-FDG. ${ }^{18} \mathrm{~F}$-fluoride ion PET/CT images were ineffective in characterizing

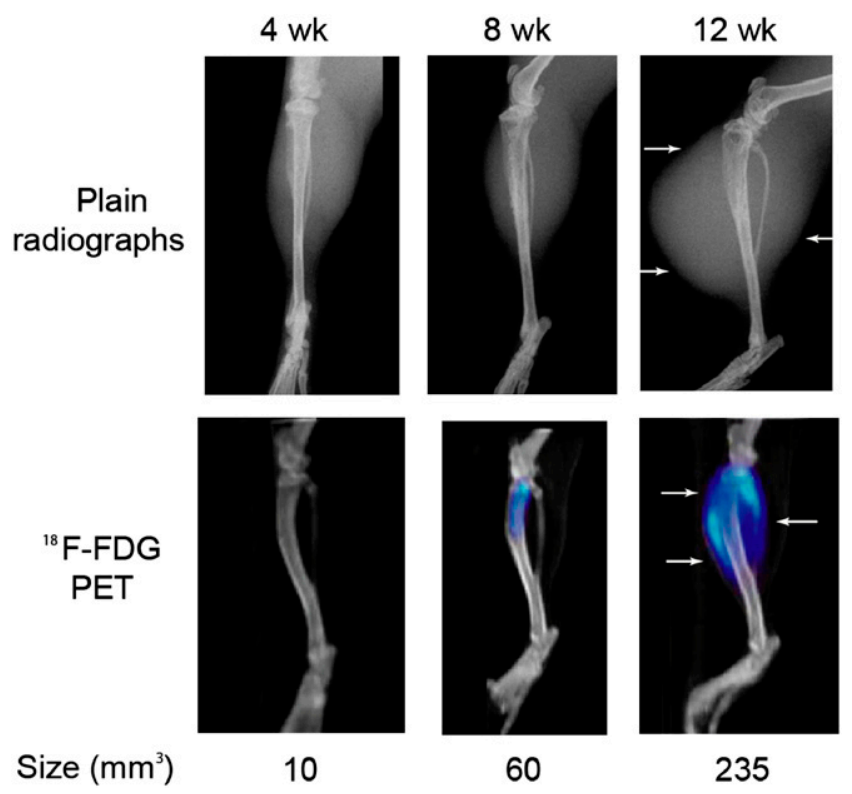

FIGURE 7. Plain radiographs and corresponding PET/CT images of a representative C42B tumor using ${ }^{18} \mathrm{~F}-\mathrm{FDG}$ at $4-$, $8-$, and $12-$ wk time points. Although an increase of ${ }^{18} \mathrm{~F}-\mathrm{FDG}$ uptake on PET images is seen on successive PET/CT images, the region of signal intensity was not found to closely approximate the large soft-tissue lesion, especially at the 12-wk time point (arrows). Quantitative analysis was used to assess the ROI (measured in $\mathrm{mm}^{3}$ and labeled below each corresponding tumor); however, these calculations consistently underestimated the soft-tissue lesion size based on tumor burden measurements. 
tumors induced by PC-3 cells because of the extensive bone loss associated with these cells. Because ${ }^{18} \mathrm{~F}$-fluoride ion uptake predominantly occurs in bone, large osteolytic lesions prohibit tracer uptake in vivo.

C42B cells typically induce a mixed osteoblastic and osteolytic lesion; however, the ${ }^{18} \mathrm{~F}$-fluoride scans detected minimal osteoblastic activity, and the soft-tissue lesions measured with ${ }^{18}$ F-FDG were smaller than direct soft-tissue burden measurements. One possible explanation for this finding is that there is a canceling effect from the overall osteoblastic and osteolytic activity of these cells. Furthermore, the smaller soft-tissue lesions measured on ${ }^{18} \mathrm{~F}-\mathrm{FDG}$ scans may be secondary to tumor cell necrosis. Cells undergoing apoptosis do not exhibit metabolic activity, which would lead to decreased radioactive tracer uptake. Necrosis was noted in the histologic specimens, and other investigators have noted high cell necrosis rates with LNCaP tumors (38). To date, this has not been investigated, and further study is necessary-possibly involving more specific radioactive probes, such as ${ }^{18} \mathrm{~F}-3^{\prime}$-deoxy-3'-fluorothymidine $\left({ }^{18} \mathrm{FLT}\right)$, which identifies areas of mitosis and proliferation (39), and ${ }^{64} \mathrm{Cu}$-ATSM (copper-diacetyl-bisN4-methylthiosemicarbazone) as an indicator of local blood flow (40).

This study suggests several potential advantages for the use of microPET/CT, including: the ability to detect an osteoblastic lesion at an earlier time point than seen on plain radiographs, to quantify the size of bone loss associated with osteolytic lesions, and to follow the biologic response to a tumor over time. However, further studies assessing the response to treatment regimens are necessary to determine whether the time and expense of microPET/CT scans provides more useful data than seen with the combination of plain radiographs, histologic and histomorphometric analysis, and CT scans.

Despite the potential advantages of molecular imaging techniques, there are certain limitations in its use for routine evaluation of small-animal prostate cancer models. First, although meticulous efforts are made to standardize and control for variations in tracer uptake within a study group, the image quality is ultimately dependent on an intravenous injection and the kidney clearance of each individual animal, which may be quite variable. Second, because the use of molecular imaging in small animals in longitudinal studies is in its relative infancy (41), the longterm effects of repeated radiation exposures to tumor growth are unknown. Although radiation doses delivered to the soft tissues from individual microCT and microPET scans have been demonstrated to be relatively low (41), outcomes from cumulative exposure from imaging at multiple time points and different modalities have yet to be studied. The cumulative dose from the imaging protocols in this study (microPET ${ }^{18} \mathrm{~F}-\mathrm{FDG}$, microPET ${ }^{18} \mathrm{~F}$-fluoride, and microCT) at each time point was $\sim 30 \mathrm{cGy}$ in the soft tissue and $\sim 60 \mathrm{cGy}$ in the bone marrow. Although these dose levels are certainly not insignificant, they compare favor- ably with the estimated median lethal dose for a single time-point radiation exposure of a mouse (8 Gy). Furthermore, in unpublished data from our laboratory, no significant effects on tumor cell growth have been seen using microPET/CT in either control or treatment groups.

Third, fluoride ion scans may have limited value when analyzing rapidly growing osteolytic lesions, as decreased tumor activity was demonstrated consistently with increasing bone loss. Again, further studies must be performed to determine whether subtle differences in treatment response can be noted with molecular imaging. Finally, the capability of microPET/CT to fully evaluate mixed lesions appears to be limited by the interaction between the osteolytic and osteoblastic components.

\section{CONCLUSION}

The advent of high-resolution molecular imaging has introduced a noninvasive, effective instrument to monitor in vivo tumor behavior and biologic interactions between itself and host tissue. In this study, we successfully used ${ }^{18} \mathrm{~F}$-fluoride ion and ${ }^{18} \mathrm{~F}$-FDG radioactive tracers to localize and quantify skeletal metabolic activity in a preclinical model of prostate cancer bone metastases. However, the true value of this technology hinges on whether subtle responses to novel treatment regimens-which cannot be elucidated with plain radiographs, histomorphometry, and histologic analysis-can be delineated with molecular imaging. Our future plan is to determine whether microPET/CT can facilitate the detection of differences in the response to new treatment modalities in animal models of metastatic disease.

\section{ACKNOWLEDGMENT}

This research was supported in part by NIH grant R24 CA92865.

\section{REFERENCES}

1. Landis SH, Murray T, Bolden S, Wingo PA. Cancer statistics, 1999. CA Cancer J Clin. 1999;49(1):8-31.

2. Koutsilieris M, Rabbani SA, Bennett HP, Goltzman D. Characteristics of prostate-derived growth factors for cells of the osteoblast phenotype. J Clin Invest. 1987;80:941-946.

3. Lange PH, Vessella RL. Mechanisms, hypotheses and questions regarding prostate cancer micrometastases to bone. Cancer Metastasis Rev. 1998;17:331336.

4. Kasper S, Smith JA Jr. Genetically modified mice and their use in developing therapeutic strategies for prostate cancer. J Urol. 2004;172:12-19.

5. Rosol TJ, Tannehill-Gregg SH, LeRoy BE, Mandl S, Contag CH. Animal models of bone metastasis. Cancer. 2003;97(3 suppl):748-757.

6. Edinger M, Sweeney TJ, Tucker AA, Olomu AB, Negrin RS, Contag CH. Noninvasive assessment of tumor cell proliferation in animal models. Neoplasia. 1999; 1:303-310.

7. Herschman HR. Micro-PET imaging and small animal models of disease. Curr Opin Immunol. 2003;15:378-384.

8. Chatziioannou AF, Cherry SR, Shao Y, et al. Performance evaluation of microPET: a high-resolution lutetium oxyorthosilicate PET scanner for animal imaging. J Nucl Med. 1999;40:1164-1175.

9. Gambhir SS, Herschman HR, Cherry SR, et al. Imaging transgene expression with radionuclide imaging technologies. Neoplasia. 2000;2:118-138. 
10. Kornblum HI, Araujo DM, Annala AJ, Tatsukawa KJ, Phelps ME, Cherry SR. In vivo imaging of neuronal activation and plasticity in the rat brain by high resolution positron emission tomography (microPET). Nat Biotechnol. 2000;18: 655-660.

11. Blau M, Nagler W, Bender MA. Fluorine-18: a new isotope for bone scanning. J Nucl Med. 1962;3:332-334.

12. Narita N, Kato K, Nakagaki H, Ohno N, Kameyama Y, Weatherell JA. Distribution of fluoride concentration in the rat's bone. Calcif Tissue Int. 1990;46: 200-204.

13. Zheng QH, Gardner TA, Raikwar S, et al. $\left[{ }^{11} \mathrm{C}\right]$ Choline as a PET biomarker for assessment of prostate cancer tumor models. Bioorg Med Chem. 2004;12:28872893.

14. Brenner W, Vernon C, Conrad EU, Eary JF. Assessment of the metabolic activity of bone grafts with ${ }^{18}$ F-fluoride PET. Eur J Nucl Med Mol Imaging. 2004;31: 1291-1298.

15. Hawkins DS, Schuetze SM, Butrynski JE, et al. $\left[{ }^{18} \mathrm{~F}\right]$ Fluorodeoxyglucose positron emission tomography predicts outcome for Ewing sarcoma family of tumors. J Clin Oncol. 2005;23:8828-8834.

16. O'Sullivan F, Roy S, O'Sullivan J, Vernon C, Eary J. Incorporation of tumor shape into an assessment of spatial heterogeneity for human sarcomas imaged with FDG-PET. Biostatistics. 2005;6:293-301.

17. Morris MJ, Akhurst T, Larson SM, et al. Fluorodeoxyglucose positron emission tomography as an outcome measure for castrate metastatic prostate cancer treated with antimicrotubule chemotherapy. Clin Cancer Res. 2005;11:32103216.

18. Oyama N, Akino H, Suzuki Y, et al. Prognostic value of 2-deoxy-2-[F-18]fluoroD-glucose positron emission tomography imaging for patients with prostate cancer. Mol Imaging Biol. 2002;4:99-104.

19. Craft N, Chhor C, Tran C, et al. Evidence for clonal outgrowth of androgenindependent prostate cancer cells from androgen-dependent tumors through a two-step process. Cancer Res. 1999;59:5030-5036.

20. Gamradt SC, Feeley BT, Liu NQ, et al. The effect of cyclooxygenase-2 (COX-2) inhibition on human prostate cancer induced osteoblastic and osteolytic lesions in bone. Anticancer Res. 2005;25(1A):107-115.

21. Whang PG, Schwarz EM, Gamradt SC, Dougall WC, Lieberman JR. The effects of RANK blockade and osteoclast depletion in a model of pure osteoblastic prostate cancer metastasis in bone. J Orthop Res. 2005;23(6): 1475-1483.

22. Lee Y, Schwarz E, Davies M, et al. Differences in the cytokine profiles associated with prostate cancer cell induced osteoblastic and osteolytic lesions in bone. J Orthop Res. 2003;21:62-72.

23. Kaighn ME, Narayan KS, Ohnuki Y, Lechner JF, Jones LW. Establishment and characterization of a human prostatic carcinoma cell line (PC-3). Invest Urol. 1979;17:16-23.

24. Wetterwald A, van der Pluijm G, Que I, et al. Optical imaging of cancer metastasis to bone marrow: a mouse model of minimal residual disease. Am J Pathol. 2002;160:1143-1153.

25. Plonowski A, Schally AV, Varga JL, et al. Potentiation of the inhibitory effect of growth hormone-releasing hormone antagonists on $\mathrm{PC}-3$ human prostate cancer by bombesin antagonists indicative of interference with both IGF and EGF pathways. Prostate. 2000;44:172-180.

26. Wieland B, Bida G, Padgett H, Go H. Current status of CTI target systems for the production of PET radiochemicals [abstract]. In: Proceedings of the 3rd Workshop on Targetry and Target Chemistry; June 19-23, 1989; Vancouver, British Columbia, Canada.

27. Hamacher K, Coenen HH, Stocklin G. Efficient stereospecific synthesis of nocarrier-added 2-[ $\left.{ }^{18} \mathrm{~F}\right]$-fluoro-2-deoxy-D-glucose using aminopolyether supported nucleophilic substitution. J Nucl Med. 1986;27:235-238.

28. Chow PL, Stout DB, Komisopoulou E, Chatziioannou AF. A method of image registration for small animal, multi-modality imaging. Phys Med Biol. 2006;51: 379-390.

29. Stout D, Chow P, Gustilo A, Grubwieser S, Chatziioannou A. Multimodality isolated bed system for mouse imaging experiments [abstract]. Mol Imaging Biol. 2003;5(suppl 3):128-129.

30. Chatziioannou A, Qi J, Moore A, et al. Comparison of 3-D maximum a posteriori and filtered backprojection algorithms for high-resolution animal imaging with microPET. IEEE Trans Med Imaging. 2000;19:507-512.

31. Loening AM, Gambhir SS. AMIDE: a free software tool for multimodality medical image analysis. Mol Imaging. 2003;2:131-137.

32. Tai C, Chatziioannou A, Siegel S, et al. Performance evaluation of the microPET P4: a PET system dedicated to animal imaging. Phys Med Biol. 2001;46:18451862.

33. Piert M, Zittel TT, Becker GA, et al. Assessment of porcine bone metabolism by dynamic. J Nucl Med. 2001;42:1091-1100.

34. Messa C, Goodman WG, Hoh CK, et al. Bone metabolic activity measured with positron emission tomography and $\left[{ }^{18} \mathrm{~F}\right]$ fluoride ion in renal osteodystrophy: correlation with bone histomorphometry. J Clin Endocrinol Metab. 1993;77: 949-955.

35. Machtens S, Boerner AR, Hofmann M, Knapp WH, Jonas U. Urologe A. 2004; 43:1397-1409.

36. Shreve PD, Grossman HB, Gross MD, Wahl RL. Metastatic prostate cancer: initial findings of PET with 2-deoxy-2-[F-18]fluoro-D-glucose. Radiology. 1996;199:751-756.

37. Salminen E, Hogg A, Binns D, Frydenberg M, Hicks R. Investigations with FDG-PET scanning in prostate cancer show limited value for clinical practice. Acta Oncol. 2002;41:425-429.

38. Bylund A, Zhang JX, Bergh A, et al. Rye bran and soy protein delay growth and increase apoptosis of human $\mathrm{LNCaP}$ prostate adenocarcinoma in nude mice. Prostate. 2000;42:304-314.

39. Shields AF, Grierson JR, Dohmen BM, et al. Imaging proliferation in vivo with [F-18]FLT and positron emission tomography. Nat Med. 1998;4:1334-1336.

40. Flower MA, Zweit J, Hall AD, et al. ${ }^{62} \mathrm{Cu}$-PTSM and PET used for the assessment of angiotensin II-induced blood flow changes in patients with colorectal liver metastases. Eur J Nucl Med. 2001;28:99-103.

41. Taschereau R, Chow PL, Chatziioannou A. Monte Carlo simulations of dose from microCT imaging procedures in a realistic mouse phantom. Med Phys. 2006;33:216-224. 